\title{
Online Cloud Offloading using Heterogeneous Enhanced Remote Radio Heads
}

\author{
Yousef N. Shnaiwer*, Sameh Sorour**, Parastoo Sadeghi ${ }^{\dagger}$, and Tareq Y. Al-Naffouri ${ }^{\ddagger}$ \\ ${ }^{*}$ King Fahd University of Petroleum and Minerals, Dhahran, Saudi Arabia \\ ** University of Idaho, Moscow, ID, USA \\ $\dagger$ The Australian National University, Canberra, Australia \\ ¥ King Abdullah University of Science and Technology, Thuwal, Saudi Arabia \\ *g201204420@kfupm.edu.sa,** samehsorour@uidaho.edu, \\ †parastoo.sadeghi@anu.edu.au, ${ }^{\ddagger}$ tareq.alnaffouri@kaust.edu.sa
}

\begin{abstract}
This paper studies the cloud offloading gains of using heterogeneous enhanced remote radio heads (eRRHs) and dual-interface clients in fog radio access networks (F-RANs). First, the cloud offloading problem is formulated as a collection of independent sets selection problem over a network coding graph, and its NP-hardness is shown. Therefore, a computationally simple online heuristic algorithm is proposed, that maximizes cloud offloading by finding an efficient schedule of coded file transmissions from the eRRHs and the cloud base station (CBS). Furthermore, a lower bound on the average number of required CBS channels to serve all clients is derived. Simulation results show that our proposed framework that uses both network coding and a heterogeneous F-RAN setting enhances cloud offloading as compared to conventional homogeneous F-RANs with network coding.
\end{abstract}

Index Terms-F-RANs, Enhanced Remote Radio Head, Network Coding, Dual Interface.

\section{INTRODUCTION}

The explosive growth in data rate demands [1] raises the need to increase the capacity of future wireless networks. Fog radio access networks (F-RANs) have emerged [2,3] as a novel prospective of $5 \mathrm{G}$ architecture that exploits the synergies between edge caching and cloud control to improve 5G throughput. Traditional F-RANs comprise a set of cacheaided wireless nodes, called enhanced remote radio heads (eRRHs), deployed by the operator and controlled by a central cloud processor to serve the clients of the mobile network.

Since the eRRHs utilize caches, the optimization of FRANs is subject to optimizing two caching problems, namely, content placement and content delivery. The content placement problem addresses the question of which files should be stored in the cache of each eRRH such that the caches' hit probability (defined as the probability that the file requested by a client is available in the cache of at least one eRRH) is maximized. On the other hand, the content delivery phase is concerned with optimizing the coding schedules of the eRRHs in order to maximize the number of served clients. These two caching problems have been addressed in the literature under different settings [4-8]. In particular, the work in [9] studied the content delivery problem in homogeneous (HOM) FRANs (i.e, F-RANs employing the cellular access technology only). The authors formulated the content delivery problem in homogeneous F-RANs as an optimization problem over an opportunistic network coding (ONC) graph. ONC exploits the diversity of clients' requests to create coded files that can be easily decoded at their respective clients, so it can be utilized to further offload clients from the cloud base station (CBS).

Nevertheless, the work in [9] did not consider two practical aspects of current wireless networks. The first aspect is the existence of two interfaces at smart devices, namely, WiFi and LTE, which can be utilized to simultaneously receive two files. The second aspect is that the wireless network could be heterogeneous (i.e., the serving wireless nodes can utilize different technologies for transmission). In this work, we consider these two practical assumption in our model. We formulate the online cloud offloading problem in heterogeneous F-RANs as an optimization problem over an ONC graph. Due to the NP-hardness of the formulated problem, a less-complex solution is needed to approach it. Therefore, we propose a simple heuristic that reduces the worst-case complexity of the solution from exponential to square law. Moreover, we derive a lower bound on the average number of required CBS channels to serve all clients. Finally we compare the online CBS offloading performance of the proposed heterogeneous F-RANs framework to that of the traditional homogeneous FRANs framework.

\section{SySTEM MODEL}

We consider a network model that consists of a set $\mathcal{U}=$ $\left\{u_{1}, \ldots, u_{U}\right\}$ of $U$ clients. Each client requests to download a subset of files from a library $\mathcal{F}=\left\{f_{1}, \ldots, f_{F}\right\}$ of $F$ files. All files in $\mathcal{F}$ are available at the cloud base station (CBS). However, a subset of files from $\mathcal{F}$ is available to an eRRH, belonging to a set $\mathcal{C}=\left\{c_{1}, \ldots, c_{C}\right\}$ of $C$ eRRHs. All eRRHs collectively possess all the files in $\mathcal{F}$. We use Has set $\mathcal{H}_{c_{i}}$ to represent the set of all stored files at eRRH $c_{i}$ and Wants set $\mathcal{W}_{u_{j}}$ to represent the set of all requested files by client $u_{j}$. To guarantee clients' QoE, each client needs to be served by at least one file in each time epoch. This implies that the clients who are not served by the eRRHs need to be served by the CBS in each time epoch.

We also assume that each client $u_{j}$ may have downloaded one or more files from $\mathcal{F}$ in previous time epochs, which 
constitute its Has set $\mathcal{H}_{u_{j}}$ at the current epoch. The eRRHs and the CBS can exploit the clients' side information to employ $\mathrm{ONC}$ and deliver the file requests in the current epoch. ONC has shown great potential to improve throughput and delay of wireless networks [10]. ONC exploits the natural diversity of received and lost files at different devices and generates coded files using XOR operations. Subsequently, the original files are retrieved at the devices by re-XORing the coded files with the previously decoded packets.

In this work, it is assumed that the clients can utilize both LTE and WiFi interfaces to receive two files simultaneously from two different eRRHs at each time epoch. We will refer to this setting as the heterogeneous F-RAN scenario. Having been low-cost devices with limited transmission capabilities, each eRRH is equipped with a single wireless card and capable of utilizing a single transmission technology. eRRHs equipped with LTE cards can utilize a single LTE physical channel (i.e., a given group of OFDMA subcarriers) that is orthogonal to the channels used by the CBS. Consequently, a client scheduled to download one of its requested files from a eRRH needs to use the same interface of that eRRH and, in case of LTE-enabled eRRHs, also needs to tune to the appropriate channel utilized by that eRRHs. The CBS, a sophisticated wireless node, is able to dynamically utilize its many orthogonal channels.

\section{Problem Formulation}

In this section, we formulate the online CBS offloading problem for heterogeneous F-RANs. We address the following question: At a given time epoch, which files should be combined and transmitted by individual eRRHs, so that the number of orthogonal CBS channels needed to serve the remaining clients is minimized?

\section{A. Dual Conflict Graph}

To formulate the online CBS offloading problem, we now define a dual conflict graph $\mathcal{G}(\mathcal{V}, \mathcal{E})$, where $\mathcal{V}$ is the set of vertices and $\mathcal{E}$ is the set of edges, for the considered heterogeneous F-RAN scenario. The dual conflict graph was first introduced in [11] and utilized in [9] for homogeneous F-RANs. Our dual conflict graph is a modification on the graph used in $[9,11]$ by taking into account dual interfaces at the clients. It is worth noting that the modified dual conflict graph represents all feasible transmissions from the eRRHs. Moreover, at a given time epoch, we remove the vertices that represent the requests of clients served by the eRRHs, and construct a CBS conflict graph using the vertices induced by the requests from the clients that are not served by any eRRH. This CBS graph is utilized to minimize the number of CBS transmissions.

For the considered network model, the eRRH's dual conflict graph is constructed as follows. Every client $u_{j}$, requesting file $f_{k}$, induces one vertex $v_{i, j, k}$ in the graph for each eRRH $c_{i}$ possessing this file. In other words, $\mathcal{V}=\left\{v_{i, j, k}\right\} \forall f_{k} \in$ $\left(\mathcal{W}_{u_{j}} \cap \mathcal{H}_{c_{i}}\right), u_{j} \in \mathcal{U}$. Two vertices $v_{i_{1}, j_{1}, k_{1}}$ and $v_{i_{2}, j_{2}, k_{2}}$ are adjacent by an edge in this graph if one of the following two conditions are true:
1) Coding conflict condition: This condition is satisfied if two vertices represent two different files, and any of these files requested by a client is not in the Has set of the other client (i.e., $f_{k_{1}} \neq f_{k_{2}}$ and either $f_{k_{1}} \notin \mathcal{H}_{u_{j_{2}}}$ OR $f_{k_{2}} \notin \mathcal{H}_{u_{j_{1}}}$ ).

2) Transmission conflict condition: This condition depends on the interfaces utilized by the eRRHs representing the two vertices:

a) If the eRRHs representing the two vertices use the same interface, the transmission conflict occurs in the case of the same client is served by two different eRRHs (i.e., $j_{1}=j_{2}$ AND $i_{1} \neq i_{2}$ ).

b) If the eRRHs representing the two vertices use two different interfaces, the transmission conflict occurs in the case of both vertices represent the same client requesting the same file served by two different eRRHs (i.e., $j_{1}=j_{2}$ AND $k_{1}=k_{2}$ AND $i_{1} \neq i_{2}$ ).

On the other hand, the CBS conflict graph can be constructed following a similar approach as the eRRH's dual conflict graph, but differing from that in the way of no transmission conflict condition. Thus, two vertices are adjacent in the CBS conflict graph if the aforementioned coding conflict condition is satisfied.

\section{B. Problem Formulation using Dual Conflict Graph}

We now use the dual conflict graph to formulate the online CBS offloading problem for heterogeneous F-RANs. Let $\mathcal{I}_{i}^{(t)}, i \in\{1, \ldots, C\}, t \in\{1, \ldots, T\}$, be the independent set that represents coded files $\mathcal{F}\left(\mathcal{I}_{i}^{(t)}\right)$ allocated to eRRH $c_{i}$ for delivery to the targeted clients in $\mathcal{U}\left(\mathcal{I}_{i}^{(t)}\right)$ in the $t$-th time epoch ${ }^{1}$. Thus, the problem boils down to finding a collection of independent sets $\left\{\mathcal{I}_{1}^{(t)}, \ldots, \mathcal{I}_{C}^{(t)}\right\}$, and minimizing the total number of independent sets in the CBS graph at the $t$-th time epoch. Moreover, the independent sets selection also needs to cover all the remaining requests that can be served by the CBS. In fact, the remaining independent sets are served by orthogonal channels at the CBS, and thus, the number of such independent sets needs to be minimized. In the $t$-th time epoch, let us define $\mathcal{J}_{M B S}^{(t)}$ as the set of CBS transmissions, $\mathcal{J}_{L T E}^{(t)}$ as the set of LTE eRRHs' transmissions and $\mathcal{J}_{W i F i}^{(t)}$ as the set of WiFi eRRHs' transmissions. At the $t$-th time epoch, the online CBS offloading problem for heterogeneous F-RANs can be formulated as:

$$
\begin{array}{ll} 
& \min _{\mathcal{I}_{1}^{(t)}, \ldots, \mathcal{I}_{C}^{(t)}} N_{M B S}^{(t)} \\
\text { s.t. } & \mathcal{F}\left(\mathcal{I}_{i}^{(t)}\right) \subseteq \mathcal{H}_{c_{i}}, \forall c_{i} \in \mathcal{C} \\
& \left|\mathcal{J}_{W i F i}^{(t)}\left(u_{j}\right)\right| \leq 1, \forall u_{j} \in \mathcal{U} \\
& \left|\mathcal{J}_{L T E}^{(t)}\left(u_{j}\right)\right| \leq 1, \forall u_{j} \in \mathcal{U} \\
& \mathcal{U}\left(\mathcal{J}_{C B S}^{(t)}\right)=\mathcal{U} \backslash \mathcal{U}\left(\mathcal{J}_{W i F i}^{(t)} \cup \mathcal{J}_{L T E}^{(t)}\right) .
\end{array}
$$

\footnotetext{
${ }^{1} \mathrm{~A}$ targeted client is able to immediately decode a new file from the received XOR file combination $\mathcal{F}\left(\mathcal{I}_{i}^{(t)}\right)$.
} 
The objective function in (1a) selects a collection of independent sets over all feasible sets of independent sets in the eRRH graph that minimizes the total number of required CBS transmissions at the $t$-th time epoch. The set of constraints in (1b) guarantees that all files allocated to an eRRH in the $t$-th time epoch are cached in that eRRH. If these cached file constraints are not satisfied for any $\mathcal{I}_{i}^{(t)}$, then an XOR of the files in $\mathcal{F}\left(\mathcal{I}_{i}^{(t)}\right)$ cannot be formed at eRRH $c_{i}$, which makes a solution including $\mathcal{I}_{i}^{(t)}$ invalid. The sets of constraints in (1c) and (1d) limit the number of vertices induced by each client at the $t$-th time epoch to one in the WiFi and LTE eRRHs' solutions, respectively. This guarantees that the number of vertices induced by each client in the eRRH solution is limited to two vertices, which represents the maximum number of files that can be received by the dual interface at a client in each time epoch. The set of constraints in (1e) defines the set of clients served by the CBS at the $t$-th time epoch as those who are not served by any eRRH.

Having formulated the minimum number of orthogonal CBS transmission problem, we now show that finding the optimal solution of this formulation is NP-hard. To prove the NPhardness, it is sufficient to consider a simple case with one request and single interface per client. The optimal solution of this single time epoch problem is equivalent to finding the chromatic number of the CBS graph [9], which is NPhard [12]. Hence, the optimization problem in (1) is an NPhard problem. Therefore, to solve the problem in (1) with much lower computational complexity, we develop a simple heuristic algorithm that efficiently reduces the required number of orthogonal CBS transmissions.

\section{The Proposed Greedy HeuristiC}

In this section, we propose a low-complexity algorithm to heuristically solve the problem in (1). Our proposed heuristic is based on the idea of decomposing the problem in (1) into two sub-problems, namely, the problem of finding the weighted maximum independent set in the CBS dual conflict graph and the problem of finding the weighted minimum graph coloring in the CBS conflict graph. Having described the dual conflict eRRH graph in Section III-A, at a given time epoch, our proposed heuristic for heterogeneous F-RANs is implemented at the CBS as shown in Algorithm 1. According to Section II, only the clients in the second group (clients who are not served by any eRRH) can be served by the CBS in order to serve every client with at least one new file. Therefore, only the clients in the second group are considered in the third step of Algorithm 1.

As we mentioned at the beginning of this section, our heuristic solves two main problems, namely, the weighted maximum independent set selection problem in the eRRHs' dual conflict graph and the weighted minimum graph coloring problem in the CBS graph. However, both problems are well known NP-hard problems [13].

To further reduce the computational complexities, we solve the aforementioned two problems at each time epoch using

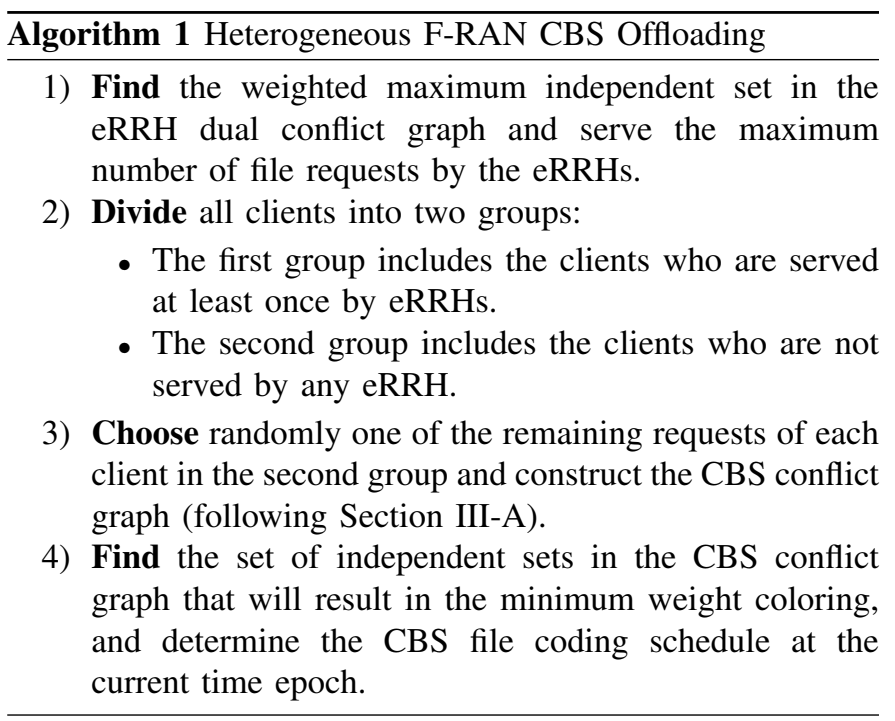

a modified version of the maximum weight vertex search algorithm proposed in [9] for homogeneous F-RANs considering erasure-free channels. For every client, the CBS assigns a counter which is initially set to zero. Each time a vertex induced by a client $u_{j}$ is chosen as part of the solution, the counter of that client is incremented by one. To find the eRRHs' solution, we define a weight $w_{i, j, k}$ for a vertex $v_{i, j, k}$ in the dual conflict eRRH graph as follows:

$$
w_{i, j, k}=\left(V-\delta_{i, j, k}\right) \times \sum_{v_{i^{\prime}, j^{\prime}, k^{\prime}} \in \mathcal{N}\left(v_{i, j, k}\right)}\left(V-\delta_{i^{\prime}, j^{\prime}, k^{\prime}}\right),
$$

where $V$ is the total number of vertices in the graph, $\delta_{i, j, k}$ is the degree of vertex $v_{i, j, k}$, and $\mathcal{N}\left(v_{i, j, k}\right)$ is the set of its adjacent vertices ${ }^{2}$.

According to (2), a vertex has a high weight when it is non-adjacent to a large number of vertices and each of these non-adjacent vertices is also non-adjacent to a large number of other vertices. Thus, a maximum weight vertex search can be performed by selecting the vertex with the highest weight. Moreover, the algorithm evaluates whether this vertex can be part of a valid solution (i.e., it satisfies the constraints in (1c) and (1d)) by examining whether the counter of the client inducing that vertex surpasses the limit of two. If it surpasses the limit, the MBS ignores this vertex and searches for the vertex with the second highest weight. Otherwise, this vertex is chosen as part of the solution and all of its adjacent vertices are removed from the graph. This process is repeated until no vertices remain in the dual conflict eRRH graph. This heuristic is also applied to the CBS graph to iteratively find the weighted maximum independent set and then remove its vertices from the CBS graph until all vertices are removed.

Now, we comment on the complexity of the proposed online CBS offloading algorithm. The basic operation in the maximum weight vertex search algorithm is the operation of comparing the weights of two different vertices. In the worst case, each pair of vertices is compared before finding the

\footnotetext{
${ }^{2}$ The degree of a vertex in a graph is the number of vertices adjacent to this vertex.
} 
solution for a given time epoch. This results in the worst case computational complexity of our algorithm which is $O\left(V^{2}\right)$.

\section{Lower Bound on the Average Number of REQUIRED CBS CHANNELS}

In this section, we derive a lower bound on the average number of required CBS channels to serve every client at least once at a given time epoch. The files are assumed to be distributed among the eRRHs using systematic fixed file placement [14].

Theorem 1: Assuming systematic fixed file placement is utilized to distribute the files among the eRRHs, the average number of CBS channels $N_{C B S}^{(t)}$ needed to serve every client at least once during the $t$-th time epoch satisfies

$$
\begin{aligned}
& N_{C B S}^{(t)} \geq\left(\frac{1}{2}+o_{1}(1)\right) \times \\
& \log \left(\frac{\left(U^{(t)}-N_{e}^{(t)}\right) F-1}{\left(\left(U^{(t)}-N_{e}^{(t)}\right) F-1\right)-\left(1-\sigma_{u}^{2}\right)\left(U^{(t)}-N_{e}^{(t)}\right)(F-1)}\right) \\
& \times \frac{U^{(t)}-N_{e}^{(t)}}{\log \left(U^{(t)}-N_{e}^{(t)}\right)}, \\
& \text { where } \quad N_{e}^{(t)}=2 \log \frac{1}{1-\tilde{\pi}^{(t)}}\left(\frac{e \tilde{\nu}^{(t)}}{2 \log \frac{1}{1-\tilde{\pi}^{(t)}} \tilde{\nu}^{(t)}}\right)+1+o_{1}(1), \\
& \text { where } \tilde{\nu}^{(t)}=(|\mathcal{W}|-(t-1)) U^{(t)} \sigma_{c} C \text {, } \\
& \tilde{\pi}^{(t)}= \\
& \frac{R(R-1)}{\tilde{\tilde{\nu}}^{(t)}\left(\tilde{\nu}^{(t)}-1\right)} P_{e}+\frac{U^{(t)}\left(U^{(t)}-1\right)\left(H_{c}-1\right)}{\left(U^{(t)} C-1\right)\left(U^{(t)} H_{c}-1\right)}(1-\Psi), \\
& \text { where } P_{e}=\left(\frac{C_{W i F i}}{C_{W i F i}+C_{L T E}}\right)\left(\frac{C_{W i F i}-1}{C_{W i F i}+C_{L T E}-1}\right)+ \\
& \left(\frac{C_{L T E}}{C_{W i F i}+C_{L T E}}\right)\left(\frac{C_{L T E}-1}{C_{W i F i}+C_{L T E}-1}\right) \text {, } \\
& \text { and } \Psi= \\
& \sum_{e_{1}^{\prime}=0}^{H_{u}} \frac{\left(\begin{array}{c}
H_{u} \\
e_{1}^{\prime}
\end{array}\right)\left(\begin{array}{c}
F-H_{u} \\
H_{u}-e_{1}^{\prime}
\end{array}\right)}{\left(\begin{array}{c}
F \\
H_{u}
\end{array}\right)} \sum_{e_{2}^{\prime}=\max \left(0, H_{u}-\left(F-H_{c}\right)\right)}^{\min \left(H_{u}-e_{1}^{\prime}, H_{c}\right)} \frac{\left(\begin{array}{c}
H_{c} \\
e_{2}^{\prime}
\end{array}\right)\left(\begin{array}{c}
F-H_{c} \\
H_{u}-e_{1}^{\prime}-e_{2}^{\prime}
\end{array}\right)}{\left(\begin{array}{c}
F \\
H_{u}-e_{1}^{\prime}
\end{array}\right)} \times \\
& \sum_{e_{3}^{\prime}=\max \left(0, H_{u}-\left(F-\left(H_{c}-e_{2}^{\prime}\right)\right)\right)}^{\min \left(H_{u}-e_{1}^{\prime}, H_{c}-e_{2}^{\prime}\right)} \frac{\left(\begin{array}{c}
H_{c}-e_{2}^{\prime} \\
e_{3}^{\prime}
\end{array}\right)\left(\begin{array}{c}
F-\left(H_{c}-e_{2}^{\prime}\right) \\
H_{u}-e_{1}^{\prime}-e_{3}^{\prime}
\end{array}\right)}{\left(\begin{array}{c}
F \\
H_{u}-e_{1}^{\prime}
\end{array}\right)} \frac{e_{2}^{\prime}}{H_{c}} \frac{e_{3}^{\prime}}{H_{c}-1},
\end{aligned}
$$

where $o_{1}(1)$ and $o_{2}(1)$ are constants between 0 and 1 that can be found empirically, $\sigma_{u}$ is the download ratio of each client (defined as the ratio of the number of files downloaded by the client to the total number of files in the library), $\sigma_{c}$ is the eRRH caching ratio, $R=\frac{C H_{c}}{F}$ is the repetition index denoting the number of copies of each file in the union of all the eRRHs' caches, where $H_{c}$ is the number of files stored in each eRRH; $C_{W i F i}$ and $C_{L T E}$ denote the number of WiFi and LTE eRRHs, respectively.

Proof: The reader is referred to Appendix A for a proof of this theorem.

\section{Vi. Simulation Results}

In this section, we present the simulation results to evaluate the performance of our proposed heuristic solution for heterogeneous F-RANs. We compare the proposed heuristic to the

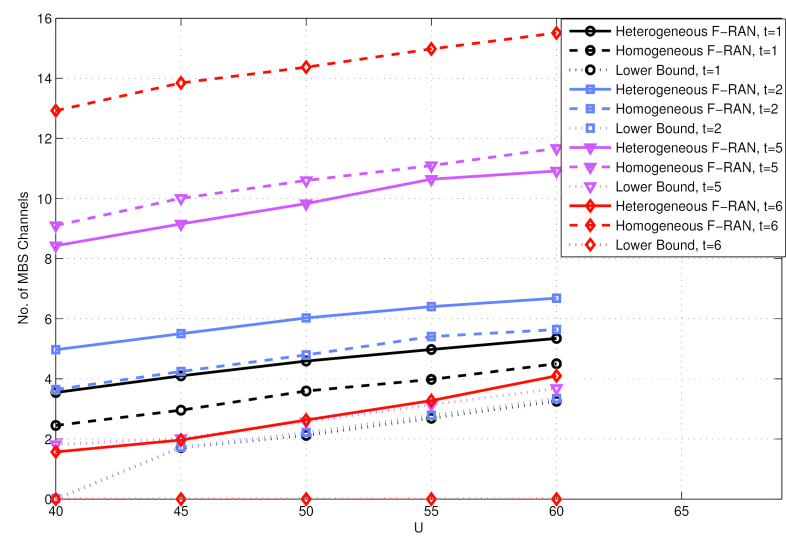

Fig. 1: Average number of required CBS channels per time epoch of heterogeneous and homogeneous F-RANs and the lower bound in (3)

homogeneous F-RAN solution that was proposed in [9]. The simulated network model comprises 5 eRRHs ( $2 \mathrm{WiFi}$ and 3 LTE eRRHs), 30 files, and 6 files wanted by each client. Each client has prior knowledge of 1 file, and each eRRH stores 18 files.

Fig. 1 compares heterogeneous F-RANs to homogeneous F-RANs and the lower bound in (3) for several time epochs. First, we observe that the lower bound in (3) is tighter for earlier time epochs, especially with the increase of the number of clients. This is due to the fact that the derived lower bound does not consider the changes in the network parameters after each time epoch. Furthermore, it can be noted that for the first and the second time epochs, the performance of homogeneous F-RANs surpasses heterogeneous F-RANs. This can be justified by recalling that heterogeneous F-RANs allow two eRRHs to serve the same client simultaneously. Even though the eRRHs are expected to serve more requests in heterogeneous F-RANs (due to smaller number of edges in the eRRH dual conflict graph), the actual number of clients served by the eRRHs is smaller. Thus the CBS has more remaining clients to serve at the first several time epochs. However, in late time epochs, the remaining number of clients to be served by the CBS is significantly reduced due to the extra served requests by eRRHs in early time epochs in heterogeneous FRANs. Thus, as we approach the last time epoch, the CBS offloading performance of heterogeneous F-RANs becomes better than that of homogeneous F-RANs, as shown in the figure.

This trait of eliminating extra requests at early time epochs results in a better aggregate offloading performance (i.e., the summation of the required number of CBS channels over all time epochs) for heterogeneous F-RANs. Fig. 2 shows an improvement of about $17 \%$ in offloading performance for heterogeneous F-RANs as compared to homogeneous FRANs. Hence, heterogeneous F-RANs are more suitable for applications where the clients can request multiple files. For such applications, using heterogeneous F-RANs results in either reducing the total number of time epochs needed to 


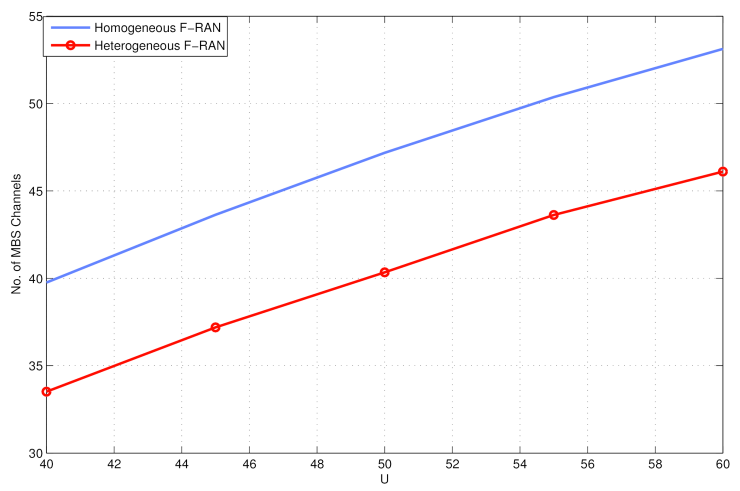

Fig. 2: Average aggregate CBS offloading performance of heterogeneous and homogeneous F-RANs over all time epochs.

serve all requests or the total number of CBS channels needed to satisfy all requests or both these cases.

\section{CONCLUSIONS}

In this paper, we investigated the effect of utilizing heterogeneous F-RAN systems on the CBS offloading performance in next generation cellular networks. For such networks, we showed that the online CBS offloading problem is NP-hard. Therefore, we proposed a low-complexity online heuristic that offloads clients using eRRHs first, then serves the remaining clients by the CBS. Furthermore, we derived a lower bound on the average number of needed CBS channels to serve all clients. Simulation results showed that the proposed heterogeneous F-RAN system results in a significant enhancement in the aggregate CBS offloading performance when compared to the traditional homogeneous F-RANs. Future extensions of this work is to address the aggregate CBS offloading problem, and to consider channel erasures in the formulation.

\section{APPENDIX A \\ ProOF OF THEOREM 1}

The online CBS offloading problem in (1) is a constrained coloring problem; and the constraint is the number of vertices induced by each client in the eRRHs' solution. Thus, a lower bound on the average number of needed CBS channels per time epoch attained using Algorithm 1 can be found by relaxing the constraints in (1c) and (1d). When the constraints are relaxed, the problem becomes a traditional coloring problem, which can be solved by finding the chromatic number of the CBS conflict graph. Using random graph theory, the chromatic number of a graph $\mathcal{G}$ can be approximated as [15]

$$
\chi(\mathcal{G})=\left(\frac{1}{2}+o_{1}(1)\right) \log \left(\frac{1}{1-\pi}\right) \frac{\nu}{\log (\nu)},
$$

where $\nu$ is the number of vertices in $\mathcal{G}$, and $\pi$ is the probability that two vertices are in conflict in $\mathcal{G}$. At a given time epoch $t$, the minimum number of vertices in the CBS graph is found by subtracting the number of requests served by the eRRHs from the total number of clients (i.e., $\nu^{(t)}=U^{(t)}-N_{e}^{(t)}$, where
$U^{(t)}$ is the number of clients requesting at least one file in the $t$-th time epoch, and $N_{e}^{(t)}$ is the number of requests served by the eRRHs in the $t$-th time epoch. $N_{e}^{(t)}$ and $\pi$ can be derived in a similar fashion to the proofs in [14].

\section{REFERENCES}

[1] "Cisco visual networking index: Forecast and methodology, 2014-2019," May 2015, White Paper.

[2] Q. Li, H. Niu, A. Papathanassiou, and G. Wu, "Edge cloud and underlay networks: Empowering 5g cell-less wireless architecture," in European Wireless 2014; 20th European Wireless Conference; Proceedings of, May 2014, pp. 1-6.

[3] S. H. Park, O. Simeone, and S. Shamai, "Joint optimization of cloud and edge processing for fog radio access networks," in 2016 IEEE International Symposium on Information Theory (ISIT), July 2016, pp. 315-319.

[4] K. Shanmugam, N. Golrezaei, A. Dimakis, A. Molisch, and G. Caire, "Femtocaching: Wireless content delivery through distributed caching helpers," IEEE Transactions on Information Theory, vol. 59, no. 12, pp. 8402-8413, Dec. 2013.

[5] M. Maddah-Ali and U. Niesen, "Fundamental limits of caching," IEEE Transactions on Information Theory, vol. 60, no. 5, pp. 2856-2867, May 2014.

[6] _ - "Decentralized coded caching attains order-optimal memory-rate tradeoff," IEEE/ACM Transactions on Networking, vol. PP, no. 99, pp. 1-1, Apr. 2014.

[7] R. Pedarsani, M. Maddah-Ali, and U. Niesen, "Online coded caching," in 2014 IEEE International Conference on Communications (ICC), June 2014, pp. $1878-1883$.

[8] N. Karamchandani, U. Niesen, M. Maddah-Ali, and S. Diggavi, "Hierarchical coded caching," in IEEE International Symposium on Information Theory (ISIT), 2014, June 2014, pp. 2142-2146.

[9] Y. N. Shnaiwer, S. Sorour, N. Aboutorab, P. Sadeghi, and T. Y. AlNaffouri, "Network-coded content delivery in femtocaching-assisted cellular networks," in 2015 IEEE Global Communications Conference (GLOBECOM), Dec 2014, pp. 1-6.

[10] W. Chen, K. B. Letaief, and Z. Cao, "Opportunistic network coding for wireless networks," in 2007 IEEE International Conference on Communications, June 2007, pp. 4634-4639.

[11] A. Al-Habob, S. Sorour, N. Aboutorab, and P. Sadeghi, "Conflict free network coding for distributed storage networks," in 2015 IEEE International Conference on Communications (ICC), June 2015, pp. $5517-5522$.

[12] C. Edwards and C. Elphick, "Lower bounds for the clique and the chromatic numbers of a graph," Discrete Applied Mathematics, vol. 5, no. 1, pp. 51 - 64, 1983. [Online]. Available: http://www.sciencedirect.com/science/article/pii/0166218X8390015X

[13] M. R. Garey and D. S. Johnson, Computers and Intractability: A Guide to the Theory of NP-Completeness. New York, NY, USA: W. H. Freeman \& Co., 1979.

[14] Y. Shnaiwer, S. Sorour, P. Sadeghi, N. Aboutorab, and T. Y. Al-Naffouri, "Network-coded macrocell offloading in femtocaching-assisted cellular networks." [Online]. Available: http://arxiv.org/abs/1606.05768

[15] B. Bollobas, "The chromatic number of random graphs," Combinatorica, vol. 8, no. 1, pp. 49-55, 1988. [Online]. Available: http://dx.doi.org/10.1007/BF02122551 